\title{
Composición Digital en la Producción Científica de Libros en la Universidad Técnica del Norte, Ecuador
}

\author{
Andrea V. Basantes, Adriano R. Carrascal, Miguel E. Naranjo y Carlos I. Almeida \\ Universidad Técnica del Norte, Campus. Avenida 17 de Julio, 5-21, General José María Córdova, Código \\ postal 100105, Ibarra, Ecuador. (e-mail: avbasantes@utn.edu.ec, arcarrascal@utn.edu.ec, \\ menaranjo@utn.edu.ec, cialmeida@utn.edu.ec)
}

Recibido Ene. 23, 2018; Aceptado Abr. 4, 2018; Versión final Jun. 8, 2018, Publicado Dic. 2018

\begin{abstract}
Resumen
El objetivo de esta investigación fue determinar el nivel de conocimiento y uso de herramientas digitales para la composición digital de libros como parte de la producción científica de la Universidad Técnica del Norte en Ecuador. Mediante un enfoque cuanti - cualitativo de corte descriptivo, bibliográfico y de campo se buscó identificar la importancia de la composición digital, el número de libros realizados y publicados por los docentes, las preferencias en formato impreso o digital, las complicaciones de la publicación, las herramientas que usan para diagramar sus libros. Se determina y analiza también el interés que tienen los docentes en actualizar y perfeccionar sus conocimientos sobre la composición digital de libros. Los resultados ponen en manifiesto que de los 103 docentes que han escrito libros el 40\% tuvo complicaciones en la publicación de sus obras por el desconocimiento del proceso de maquetación, el $29 \%$ por el proceso de publicación y el $31 \%$ se debe a otros factores. En conclusión, se evidencia el desconocimiento de herramientas de composición digital de libros, haciéndose necesaria la implementación de un plan de capacitación sobre maquetación.
\end{abstract}

\section{Digital Composition in the Scientific Production of Books at the Universidad Técnica del Norte, Ecuador}

\begin{abstract}
The objective of this research was to determine digital tools knowledge and usage for digital compositing of books as part of the scientific production at the Universidad Técnica del Norte in Ecuador. A qualitative descriptive, bibliographic and field approach was used to identify the importance of digital compositing, number of books written and published by professors, preferences in printed or digital format, publishing complications, and tools used to diagram their books. Also, professors' interests in updating and increasing their knowledge about digital compositing of books is determined and analyzed. The results show that of the 103 professors who have written books, $40 \%$ had complications in their work publishing due to the lack of knowledge about layout process, $29 \%$ due to the publishing process, and 31\% due to other reasons. In conclusion, the lack of knowledge about digital book compositing tools is evident, being necessary the implementation of a training plan on book layout.
\end{abstract}

Keywords: digital composition; tools; layout; books; scientific production 


\section{INTRODUCCIÓN}

En los últimos años las Instituciones de Educación Superior (IES) se han visto inmersas en diversos procesos de evaluación que buscan analizar la productividad científica de las universidades. Actualmente, la competitividad global de las IES tiene mayor relevancia y se esfuerzan por lograr una posición superior en la jerarquización de los rankings universitarios (Zornic, Bornmann, Maricic, Markovic, Martic y Jeremic, 2015). En una época de expansión educativa y de interconexión global, los rankings internacionales en la educación superior son un componente y un estímulo importante en esta competencia posicional para lograr el estatus de "clase mundial" (Enders, 2015). El lugar que ocupa cada institución en los rankings internacionales depende de una serie de indicadores (académicos - financieros) y de su ponderación relativamente diferenciada; sin embargo, el informe final del ranking genera repercusiones en las IES en el ámbito social, académico y económico que puede ser positivo como negativo.

Autores como Pérez (2015) y Ordorika (2015) coinciden que, los rankings internacionales más destacados son: el Times Higher Education World University Ranking (THE), el Webometrics, el QS World University Ranking (QS) y el Academic Ranking of World Universities (ARWU) de la Universidad de Shanghái Jiao Tong (más conocido como Ranking de Shanghái), éste último con mayor influencia en la actualidad, el cual clasifica a las instituciones según el rendimiento académico o de investigación (Martínez, 2001). Los indicadores que emplea ARWU para establecer el ranking hacen referencia a los premios internacionales, investigadores altamente citados, artículos publicados en revistas principales seleccionadas y/o indexados por índices de citas y el rendimiento per cápita (Nian y Ying, 2011). La visibilidad de las universidades ecuatorianas en los rankings internacionales es escasa, en el Ranking de Shanghái del 2018 no hay ninguna universidad ecuatoriana en los primeros 500 puestos. En los 1000 primeros puestos apenas aparecen dos universidades ecuatorianas: Universidad San Francisco de Quito (701-750) y la Pontificia Universidad Católica del Ecuador (801-1000). Los informes de los rankings universitarios han sido objetos de controversia y debate sobre: la capacidad efectiva de este tipo de instrumentos para valorar la calidad de las instituciones de educación superior, las variables consideradas, la metodología de cálculo, la subjetividad de los indicadores entre otros (Albornoz y Osorio, 2017); sin embargo, esto no ha evitado que los rankings desarrollen estrategias para mejorar su competitividad y sustentabilidad en el tiempo, con un elevado grado de legitimidad y credibilidad en el campo institucional de las universidades, convirtiéndose en referentes de excelencia y calidad (Dávila, 2018). En este sentido, varios países han implementado políticas y herramientas de evaluación institucional a fin de establecer y ejecutar planes de mejora en la calidad universitaria nacional que mejoren sus resultados y posiciones en este ranking.

En Ecuador, el Consejo de Evaluación, Acreditación y Aseguramiento de la Calidad de la Educación Superior (CEAACES, 2015) realiza la evaluación de las Instituciones de Educación Superior (IES), los criterios definidos para la evaluación son: infraestructura, academia, eficiencia académica, investigación y organización. En el ámbito de investigación se consideran cuatro indicadores: planificación de la investigación, investigación regional, producción científica y libros revisados por pares; estos permiten determinar el nivel que las IES han alcanzado en la generación de un nuevo conocimiento a través de la investigación científica. La sociedad del conocimiento/información se caracteriza, entre otros aspectos, por el rápido incremento en la producción de conocimiento, los procesos de innovación, el uso creciente y sistemático de nuevas tecnologías (Basantes, Naranjo, Gallegos y Benítez, 2017); la producción científica evoluciona de manera sistemática y en este proceso se incorporan nuevas estrategias que promueve mayores niveles de participación, interacción y conformación de redes colaborativas de aprendizaje (Aithal, 2016).

Las universidades constituyen los lugares más efectivos para realizar investigación (Altbach, 2009), estas se configuran como un elemento estructural de desarrollo del conocimiento, encargadas de la formación humana que contribuyen de manera significativa a la investigación, el desarrollo y la innovación (Rodríguez-Ponce et al., 2014). Si bien las funciones de docencia, investigación, gestión y vinculación se consideran propias de la tarea universitaria, en el Ecuador se ha puesto mayor énfasis en la necesidad de perfeccionar las dos primeras debido al rol relevante que ejerce en la competitividad del país. El desarrollo de la producción científica favorece la actualización y perfeccionamiento constante desde la praxis docente, genera nuevos contextos de transferencia y aplicación del conocimiento que permiten dar solución a las necesidades sociales, culturales, políticas, educativas, entre otras.

Es un hecho que el desarrollo de la actividad científica de las universidades requiere un significativo aporte financiero de los estados debido al papel central que cumple la investigación dentro del desarrollo económico (Wang, Wang y Liu, 2011), con este aporte, las instituciones de educación superior divulgan a la sociedad el conocimiento por medio de libros, artículos científicos, ponencias, entre otros. Esta producción científica es una forma tangible y real de medir la experiencia científica y la competencia en investigación, este se convierte en un indicador valioso dentro del proceso de acreditación universitaria a nivel nacional y se transforma en presupuesto que financia otras investigaciones que permiten calificar mejor a las IES en los rankings 
nacionales e internacionales; por lo tanto, la producción científica es una función sustancial de la investigación y esta de la universidad.

La Ley Orgánica de Educación Superior (LOES, 2010), en el artículo 8, literal a), estimula a que la educación superior desarrolle el pensamiento, genere producción científica y promocione la transferencia e innovación tecnológica. En los últimos años las IES han incorporado mecanismos de financiamiento que incentivan el diseño y ejecución de proyectos investigativos, cuyos resultados son divulgados mediante artículos científicos, libros, capítulos de libro, ponencias entre otros. El incremento de la actividad investigativa en la Universidad Técnica del Norte se refleja en el número de publicaciones adscritas a la institución. Sin embargo, los índices en el área investigativa aún no cumplen las expectativas deseadas; por lo tanto, la institución hace esfuerzos importantes para que la producción científica producida por los docentes sea publicada a pesar de la crisis económica que atraviesa el país (Lebeau et al., 2012), el estado ecuatoriano y las IES.

La producción del conocimiento debe considerarse como el patrimonio de la universidad en beneficio para la sociedad; el propósito de la universidad del presente y futuro será entendido como la articulación de tres aspectos: la educación, la investigación e impulso a la difusión y el uso del conocimiento (Lux y Pérez, 2017). La metodología del trabajo docente ya no responde a la individualidad sino al trabajo colaborativo, a la conformación de grupos de investigación locales, nacionales e internacionales, inter y multidisciplinares, que a través de sus aportes permitan cubrir los intereses y alcanzar objetivos de cada proyecto (Torres-Velandia y Jaimes-Cruz, 2015). Para lograr esto, es necesario gestionar los procesos dentro de las redes académicas y fomentar el desarrollo de búsqueda, innovación y educación (Valencia y Cázares, 2016).

Para los docentes la publicación científica de artículos científicos, libros, capítulos de libro cobra mayor importancia dentro del currículum profesional; el número y la calidad de la producción científica determina el nivel investigativo y la categorización del docente dentro de las IES. La calidad y relevancia de la producción científico-académica de los libros radica en la relación que existe con el área específica de conocimiento del docente, el registro de los créditos (autoría), filiación institucional del o los autores, sello editorial donde se publicó la obra, la cual debe ser de reconocido prestigio que garantice el proceso riguroso de selección y evaluación del trabajo, registro ISBN (International Estándar Book Number) y fundamentalmente contar con la revisión de pares externos los cuales evalúan la calidad, originalidad, factibilidad y rigor científico de la obra. Cabe señalar que, antes de la publicación se añade a la valoración de libros el aporte científico de la obra, la redacción y corrección de estilo y la diagramación, en este último punto los docentes necesitan lineamientos definidos para la correcta maquetación y publicación de sus libros.

La maquetación o composición digital se entiende como el conjunto de ideas, pensamientos, expresiones textuales, gráficas y otros elementos ordenados y sistematizados en un documento (libro, artículo u otro) (Tirado, 2016); los docentes al ignoran estas consideraciones hacen que el libro pierda calidad y provoca que el lector no comprenda lo que lee, ocasiona cansancio visual y aburrimiento. La maquetación de un libro debe favorecer la motivación y comprensión lectora, encontrar las páginas o secciones de forma inmediata y fundamentalmente revisar el cumplimiento de los estándares mínimos de publicación para que la obra sea considerada en la evaluación institucional universitaria. Por lo tanto, el objetivo principal de este estudio fue determinar el nivel de conocimiento y uso de herramientas digitales para la composición digital de libros como parte de la producción científica de la Universidad Técnica del Norte.

\section{METODOLOGÍA}

La metodología representa la forma en que el investigador recopila la información, compila, analiza, procesa, sistematiza y concluye; en este caso la metodología conllevó 4 fases: estudio y fuentes de información; población y muestra; instrumentos de la investigación y análisis de resultados.

\section{Estudio y fuentes de información}

Esta investigación tiene un enfoque cuanti - cualitativo, de corte descriptivo, bibliográfico y aplicado ya que se utilizan los hallazgos de la investigación para solucionar la problemática relacionada a la composición digital de libros como producción científica de los docentes en la Universidad Técnica del Norte. Se obtuvo información primaria al aplicar la encuesta única a los sujetos sometidos al proceso de investigación.

\section{Población y muestra}

El universo de estudio estuvo conformado por 589 docentes de la Universidad Técnica del Norte, para obtener la muestra se trabajó con un nivel de confianza del $95 \%$, para la distribución muestral en cada facultad y género se realizó una afijación proporcional, los resultados de la distribución se detallan en la Tabla1. Posteriormente, se realizó la recolección y análisis de la información secundaria contenida en diversas fuentes 
bibliográficas tales como Scopus, SCimago, Scielo, Ebsco, y ProQuest a las cuales se accedió mediante el acceso virtual de la biblioteca UTN. Mediante el análisis crítico de estos documentos se extrajo las ideas principales y secundarias más relevantes de la composición digital de libros, la importancia de la producción científica en el área de investigación de las Instituciones de Educación Superior.

Tabla 1: Distribución muestral.

\begin{tabular}{|c|c|c|c|c|c|c|}
\hline \multirow{2}{*}{ Facultad } & \multicolumn{2}{|c|}{ Hombres } & \multicolumn{2}{c|}{ Mujeres } & \multicolumn{2}{c|}{ Total } \\
\cline { 2 - 7 } & $\mathrm{f}$ & $\%$ & $\mathrm{f}$ & $\%$ & $\mathrm{f}$ & $\%$ \\
\hline FACAE & 34 & $22 \%$ & 17 & $21 \%$ & 51 & $22 \%$ \\
\hline FCCSS & 8 & $5 \%$ & 21 & $26 \%$ & 28 & $12 \%$ \\
\hline FECYT & 38 & $25 \%$ & 21 & $26 \%$ & 59 & $25 \%$ \\
\hline FICA & 45 & $29 \%$ & 8 & $10 \%$ & 53 & $23 \%$ \\
\hline FICAYA & 27 & $18 \%$ & 11 & $14 \%$ & 39 & $17 \%$ \\
\hline POSTGRADO & 2 & $1 \%$ & 2 & $3 \%$ & 4 & $2 \%$ \\
\hline Total & 154 & $100 \%$ & 80 & $100 \%$ & 234 & $100 \%$ \\
\hline
\end{tabular}

\section{Instrumentos de la investigación}

Para la validez y con la finalidad de determinar el nivel de conocimiento y uso de herramientas digitales para la composición digital de libros (diagramación o maquetación) como parte de la producción científica docente, se solicitó el apoyo de tres expertos de la Universidad Técnica del Norte en el área de Investigación y Diseño Gráfico para que validen el instrumento investigativo, los cuales evaluaron la pertinencia, estructura, redacción y claridad de cada una de las preguntas; el instrumento final se conformó con 17 preguntas de selección múltiple.Consecutivamente, se realizó la confiabilidad, debido a que el instrumento está construido con variables cualitativas nominales excluyentes discrecionales, se empleó la prueba $r$ de Pearson. Para medir la estabilidad del instrumento se aplicó una prueba piloto y se seleccionaron 44 personas completamente al azar 8 por cada facultad y 4 del Instituto de Postgrado a quienes se les aplicó el instrumento. Luego de haber contestado se dividió aleatoriamente en dos partes iguales, es decir 22 encuestas para X y 22 encuestas para Y. Según Herrera (1998) para determinar la confiabilidad del instrumento establece una escala con un límite inferior $(<0,53$ a 1$)$ y límite superior $(0,53$ a 0,99$)$ con seis categorías: confiabilidad nula, confiabilidad baja, confiable, muy confiable, excelente confiabilidad y confiabilidad perfecta.

Aplicada esta escala de confiabilidad se obtuvo el valor de 0.8226; se afirma que el instrumento corresponde a las variables cualitativas nominales y tiene una excelente confiabilidad y puede ser aplicado. Adicionalmente, el instrumento contiene variables cualitativas ordinales construidos bajo la escala Likert, en este caso fue necesario medir la consistencia interna del instrumento para determinar su confiabilidad, para ello se empleó la fórmula del Alpha de Cronbach. Se tomó aleatoriamente a 15 individuos de los 44 que participaron en la prueba piloto y que respondieron SI en la pregunta 4, los datos se presentan en la Tabla 2.

Tabla 2: Confiabilidad con Alpha de Cronbach.

\begin{tabular}{|c|c|c|c|c|c|c|c|}
\hline \multirow{2}{*}{ Género } & \multirow{2}{*}{ Facultad } & \multirow{2}{*}{ Sujetos } & \multicolumn{3}{|c|}{ Ítems } & \multirow{2}{*}{ Total } & \multirow{2}{*}{$\mathrm{T}^{\wedge} 2$} \\
\hline & & & 15 & 16 & 17 & & \\
\hline Masculino & FACAE & 1 & 4 & 4 & 4 & 12 & 144 \\
\hline Masculino & FACAE & 2 & 5 & 5 & 5 & 15 & 225 \\
\hline Femenino & FECYT & 3 & 5 & 5 & 5 & 15 & 225 \\
\hline Femenino & FECYT & 4 & 5 & 5 & 5 & 15 & 225 \\
\hline Femenino & FCSS & 5 & 5 & 4 & 5 & 14 & 196 \\
\hline Masculino & FCSS & 6 & 5 & 5 & 5 & 15 & 225 \\
\hline Femenino & FICA & 7 & 2 & 2 & 5 & 9 & 81 \\
\hline Femenino & FICAYA & 8 & 1 & 1 & 1 & 3 & 9 \\
\hline Masculino & FICAYA & 9 & 2 & 2 & 5 & 9 & 81 \\
\hline Masculino & FCSS & 10 & 4 & 3 & 3 & 10 & 100 \\
\hline Masculino & FACAE & 11 & 5 & 5 & 5 & 15 & 225 \\
\hline Femenino & FACAE & 12 & 3 & 3 & 4 & 10 & 100 \\
\hline Masculino & FECYT & 13 & 5 & 2 & 5 & 12 & 144 \\
\hline Masculino & FECYT & 14 & 3 & 1 & 3 & 7 & 49 \\
\hline Masculino & FICAYA & 15 & 2 & 2 & 4 & 8 & 64 \\
\hline & & Suma & 56 & 49 & 64 & 169 & 2093 \\
\hline & & Media & 3,73 & 3,27 & 4,27 & 11,27 & \\
\hline & & Varianza & 1,93 & 2,20 & 1,26 & 5,39 & \\
\hline
\end{tabular}


El resultado obtenido con la fórmula Alpha de Cronbach fue de 0,8585 y en la escala de Herrera (1998) se afirma que, tanto en la medida de estabilidad para las variables nominales como en la consistencia interna del instrumento de las variables ordinales se obtuvo una Excelente Confiabilidad por consiguiente con esos niveles de confiabilidad el instrumento es idóneo y puede ser aplicado, el rigor científico al que fue sometido el instrumento así lo determina. Posteriormente, se aplicó el instrumento de forma online a la muestra objeto de estudio, el cual permitió indagar el número de libros realizados y publicados por los docentes; el formato de las publicaciones y preferencias (físico o digital); las complicaciones de la publicación de sus obras; las características básicas y herramientas que usan para elaborar, editar y diagramar sus libros; finalmente el interés que tienen los docentes en actualizar y perfeccionar sus conocimientos sobre la composición digital de libros.

\section{Análisis de resultados}

Los datos obtenidos con el instrumento de investigación (cuestionario) fueron procesados en una hoja de cálculo de Microsoft Excel 2016, posteriormente se utilizó el Paquete Estadístico de Investigación Social (SPSS) donde a través del cruce de variables se logró establecer los resultados y conclusiones de la investigación.

\section{RESULTADOS Y DISCUSIÓN}

De un total de 234 docentes encuestados el 44\% han escrito libros, mientras que el 56\% no; de los 103 docentes que han escrito libros el $63 \%$ son de género masculino y el $37 \%$ son de género femenino, este hallazgo es concordante con los informes internacionales, en los que las mujeres tienen una producción científica más baja y limitada participación en investigación (McCarren y Goldman, 2012; Christianson et al., 2012). Por otro lado, 45 docentes publicaron sus libros en formato físico, mientras que 30 de ellos lo hicieron en formato físico y electrónico, seguido de 28 docentes que publicaron en formato digital. Es interesante observar que los docentes aún hacen uso limitado de los libros electrónicos como medio de divulgación científico y académico. Probablemente se deba al desconocimiento de las ventajas que conlleva publicar en este formato, cómo proteger sus derechos y qué editores lo permiten. Se cree que, a medio o largo plazo, los docentes vean en el libro electrónico un medio más efectivo de divulgación científica y académica.

Por otro lado, la redacción científica alcanza un nivel mayor según la experiencia investigativa del docente, tal es así, que el $47 \%$ con una edad comprendida entre 36 y 46 años tienen 48 publicaciones, como se evidencia en la Tabla 3. En concordancia con el resultado obtenido por Meza (2018), los docentes más jóvenes recién empiezan a ejercer su profesión, emprenden el desarrollo de la investigación y utilizan más estrategias de posicionamiento que los expertos; mientras que, los docentes con mayor edad pierden el interés de publicar porque se encuentran en la culminación de su actividad profesional.

Tabla 3: Edad, publicación de libros y formato.

\begin{tabular}{|c|c|c|c|c|c|c|c|c|}
\hline \multirow{2}{*}{ Edad } & \multicolumn{7}{|c|}{ Formato } & \multicolumn{2}{c|}{ Total } \\
\cline { 2 - 9 } & Electrónico & \multicolumn{2}{|c|}{ Físico } & Físico y electrónico & \multicolumn{2}{c|}{} \\
\cline { 2 - 9 } & $f$ & $\%$ & $f$ & $\%$ & $f$ & $\%$ & $f$ & $\%$ \\
\hline De 25 a 35 años & 9 & $32,1 \%$ & 7 & $15,6 \%$ & 8 & $26,7 \%$ & 24 & $23,3 \%$ \\
\hline De 36 a 46 años & 16 & $57,2 \%$ & 23 & $51,1 \%$ & 9 & $30,00 \%$ & 48 & $46,6 \%$ \\
\hline De 47 a 57 años & 3 & $10,7 \%$ & 13 & $28,9 \%$ & 11 & $36,6 \%$ & 27 & $26,2 \%$ \\
\hline De 58 o 68 años & 0 & $0,0 \%$ & 2 & $4,4 \%$ & 2 & $6,7 \%$ & 4 & $3,9 \%$ \\
\hline Total general & 28 & $100 \%$ & 45 & $100 \%$ & 30 & $100 \%$ & 103 & $100 \%$ \\
\hline
\end{tabular}

El $52 \%$ de los docentes tienen publicado al menos un libro, de este segmento el $78 \%$ manifiesta que sus publicaciones cuentan con el registro ISBN (International Estándar Book Number). En el Ecuador, los ISBN tanto para libros físicos como electrónicos son asignados por la Cámara Ecuatoriana del Libro, quienes sistematizan la producción editorial del país con relación al área temática, autor, edición, idioma entre otras características. El incremento de la producción científica es evidente en las estadísticas y datos ISBN de la Agencia ecuatoriana, en el 2016 se registró 5392 producciones por títulos, es decir, 821 puntos más que en el 2015 que fue de 4571 producciones.

Los docentes que registraron sus obras en el 2016, se relacionan directamente con las áreas de conocimiento de las Ciencias Sociales, lo cual se contrasta con los datos de la Cámara Ecuatoriana del Libro (2016), donde se ratifica la presencia mayoritaria de las ciencias sociales $(50,90 \%)$ en la producción editorial universitaria, seguido por Literatura y Retórica (15,91\%), Tecnología - Ciencias Aplicadas (11,44\%) y con porcentajes inferiores al $5 \%$ y en su orden descendente Ciencias Naturales y Matemáticas, Generalidades, Filosofía y Psicología, Artes Bellas y Artes Decorativas, Lenguas, Geografía e Historia y Religión. 
Es preciso señalar que, a pesar de haber escrito libros, el 16\% de los docentes no ha realizado la publicación de la obra ya sea en formato digital o físico tal como se muestra en la Tabla 4. Al no realizar la publicación de los resultados investigativos se colige que la investigación no culmina. Como docentes universitarios tienen la responsabilidad de publicar su investigación; solo así será conocida por la comunidad académica, sus resultados serán discutidos y su contribución formará parte del conocimiento científico universal. Si un investigador publica se da a conocer, sus trabajos son citados, dan origen a nuevos estudios, eleva el nivel de sus investigaciones y fortalece el prestigio de la institución a la que pertenece (Valderrama, 2005).

Tabla 4: Libros publicados por los docentes.

\begin{tabular}{|c|c|c|}
\hline Libros Publicados & $\mathrm{F}$ & $\%$ \\
\hline No Publicado & 16 & $15,5 \%$ \\
\hline Dieciséis & 1 & $1,0 \%$ \\
\hline Nueve & 1 & $1,0 \%$ \\
\hline Ocho & 3 & $2,9 \%$ \\
\hline Seis & 2 & $1,9 \%$ \\
\hline Cinco & 4 & $3,9 \%$ \\
\hline Cuatro & 5 & $4,9 \%$ \\
\hline Tres & 5 & $4,9 \%$ \\
\hline Dos & 12 & $11,7 \%$ \\
\hline Uno & 54 & $52,4 \%$ \\
\hline Total & 103 & $100 \%$ \\
\hline
\end{tabular}

Al preguntar las complicaciones que tuvieron para publicar los libros, respondieron que se debe al desconocimiento del proceso de maquetación (40\%), el proceso de publicación (29\%), la estructura de un libro (16\%). Apenas el $17 \%$ expresó no haber tenido ningún tipo de obstáculo para publicar su obra. A diferencia de otros resultados que muestran que la escasa publicación de sus libros se debe a la limitada motivación y tiempo de dedicación en las actividades de investigación (Carrillo-Vargas, 2014). Se evidencia que dentro de las políticas de evaluación y acreditación institucional no se indaga las razones de la limitada publicación de libros; solamente se cuantifica la participación docente en la publicación de sus obras. Esto debe llevar a la reflexión universitaria sobre su accionar para tomar las medidas correctivas e integrar estrategias que permita asesorar a los docentes investigadores sobre la edición de libros hasta la publicación final de la obra; asimismo parte de la problemática y respuesta se encuentra en la formación de nuevos investigadores; para ello, cada una facultad debe tener en cuenta a la investigación como eje de formación e instrumento de desarrollo institucional.

Al hacer un análisis en cada una de las unidades académicas de la Universidad Técnica del Norte se obtuvo que en Postgrado el $50 \%$ tiene problemas en el proceso de maquetación, en la FACAE el problema se manifiesta con un $45 \%$, mientras que en la FCSS el inconveniente se presenta con el 44\%, en la FICAYA el $37 \%$ y donde menos se presenta este problema es en la FECYT y FICA (33\%). Este análisis es necesario e importante al momento de planificar la capacitación docente en las diferentes unidades académicas, con la finalidad de tomar en cuenta en primer lugar a aquellas facultades en donde el problema es mayor. En consonancia con Acevedo, Montero y Duran (2016) es necesario generar planes de motivación orientados a que los docentes acepten como tarea imperativa el investigar y publicar sus conocimientos con el fin de generar una cultura de publicación en la comunidad científica.

Para confirmar el nivel de conocimiento y uso de herramientas digitales para la composición digital de libros (diagramación o maquetación) como parte de la producción científica docente se indagó sobre las herramientas que usan para elaborar sus libros, dando como resultado que el $67 \%$ de docentes utilizan el procesador de textos denominado Microsoft Word, solamente un $11 \%$ conoce Indesing, software especializado para editar texto, contenidos, organización de espacios; es decir, todo lo referente a maquetación. El docente al no ser un especialista en composición digital elabora sus libros en Microsoft Word; sin embargo, su desconocimiento sobre la maquetación de libros implica que el desarrollo de su documento en Word sea improductivo a la hora de diagramar su obra en un paquete profesional de maquetación como Indesing. El docente puede considerar algunos aspectos que le permitirá al diagramador agilizar el proceso de maquetación, estos son: el tamaño de la fuente 11012 para todo el documento, el tipo de fuente arial o times new roman, las tablas sin formato (sin color, sin negritas, con la misma tipografía del texto y con las normas de publicación que utilice en todo el documento), las figuras y fotografías deben tener mínimo 300 dpi de resolución y se recomienda que estén fuera del documento para luego insertarlas, ya que en Word se pierde la resolución, las fórmulas matemáticas deben ser insertadas como imagen y no es necesario elaborar 
el índice ya que este se genera de forma automática en la diagramación; en síntesis el formato del documento en Word debe ser sencillo, sin formato. En este sentido se corrobora que el limitado conocimiento sobre composición digital entorpece la maquetación y publicación de los libros en la institución. Ver Tabla 5.

Tabla 5: Herramienta para elaborar libros.

\begin{tabular}{|l|c|c|}
\hline \multicolumn{1}{|c|}{ Opciones } & $f$ & $\%$ \\
\hline Desconozco & 13 & $12,6 \%$ \\
\hline Indesing & 11 & $10,7 \%$ \\
\hline Indesing, PowerPoint & 1 & $1,0 \%$ \\
\hline Indesing, Word & 2 & $1,9 \%$ \\
\hline Word & 69 & $67,0 \%$ \\
\hline Word, Desconozco & 1 & $1,0 \%$ \\
\hline Word, PowerPoint & 5 & $4,9 \%$ \\
\hline Word, Quarexpress, PowerPoint & 1 & $1,0 \%$ \\
\hline Total general & 103 & $100 \%$ \\
\hline
\end{tabular}

Al preguntar si considera que los libros electrónicos llegarán a imponerse sobre los libros en papel, el criterio general de todos los sujetos sometidos al proceso de investigación con un valor ponderado de 2,56 y este valor ubicado en la categoría correspondiente (ver Tabla 6) significa que los libros electrónicos llegarán a imponer sobre los libros en papel (mucho).

Este estudio, al igual que otras investigaciones (Cordón-García, et al., 2013; Prasetya, Widiyaningtyas, Arifin, y I, 2017) evidencia que se está incrementando gradualmente el uso de los libros electrónicos en los entornos académicos y científico ya que tiene mayores niveles de distribución a nivel mundial, no se deteriora, puede ser consultado en cualquier momento a través de la red de Internet y no se circunscriben al espacio físico de la biblioteca; además está ligado al crecimiento de los dispositivos móviles (teléfonos móviles, teléfonos inteligentes (Smartphone), Tablet entre otros) debido a la demanda cada vez mayor en las diferentes actividades cotidianas.

Tabla 6: Ponderación libros electrónicos.

\begin{tabular}{|c|c|c|c|c|}
\hline Categorías & $f$ & $\%$ & $V c$ & $\%{ }^{*} V_{c}$ \\
\hline Mucho & 143 & $61,1 \%$ & 3 & 1,83 \\
\hline Poco & 78 & $33,3 \%$ & 2 & 0,67 \\
\hline Nada & 13 & $5,6 \%$ & 1 & 0,06 \\
\hline Total & 234 & $100 \%$ & $V p$ & 2,56 \\
\hline
\end{tabular}

Por otro lado, en la Tabla 7 se evidencia que la gran mayoría de los encuestados (93\%) manifiestan tener interés para capacitarse sobre la composición digital de libros lo que seguramente facilitará la publicación de sus libros y mejorará la evaluación institucional.

Tabla 7: Interés en capacitación.

\begin{tabular}{|c|c|c|}
\hline Opciones & $f$ & $\%$ \\
\hline No & 16 & $6,8 \%$ \\
\hline $\mathrm{Si}$ & 218 & $93,2 \%$ \\
\hline Total general & 234 & $100 \%$ \\
\hline
\end{tabular}

De los docentes que manifiestan interés de capacitarse sobre maquetación, el $28 \%$ pertenece a la FACAE, seguido del $25 \%$ que son de la FECYT, el $19 \%$ de la FICA, seguido por el $17 \%$ de la FICAYA. Aunque resulta paradójico que, donde tienen más inconvenientes para publicar sus libros (ver Tabla 8) no muestran mayor interés en actualizar o perfeccionar sus conocimientos sobre composición digital de libros (FSCC 10\% y Postgrado 2\%). 
Tabla 8: Interés en capacitación por facultad.

\begin{tabular}{|l|c|c|c|c|c|c|}
\hline \multirow{2}{*}{ Facultades } & \multicolumn{2}{c|}{ No } & \multicolumn{2}{c|}{ Si } & \multicolumn{2}{c|}{ Total general } \\
\cline { 2 - 7 } & $f$ & $\%$ & $f$ & $\%$ & $f$ & $\%$ \\
\hline FACAE & 2 & $12,4 \%$ & 60 & $27,5 \%$ & 62 & $27,5 \%$ \\
\hline FCSS & 0 & $0,0 \%$ & 21 & $9,6 \%$ & 21 & $9,6 \%$ \\
\hline FECYT & 5 & $31,3 \%$ & 55 & $25,3 \%$ & 60 & $25,2 \%$ \\
\hline FICA & 4 & $25,0 \%$ & 42 & $19,3 \%$ & 46 & $19,3 \%$ \\
\hline FICAYA & 5 & $31,3 \%$ & 36 & $16,5 \%$ & 41 & $16,5 \%$ \\
\hline POSTGRADO & 0 & $0,0 \%$ & 4 & $1,8 \%$ & 4 & $1,8 \%$ \\
\hline Total general & 16 & $100 \%$ & 218 & $100 \%$ & 234 & $100 \%$ \\
\hline
\end{tabular}

\section{CONCLUSIONES}

Sobre la base de los resultados de la investigación, se obtuvo las siguientes conclusiones:

1) En el contexto actual, marcado por la situación económica, las políticas de evaluación, acreditación y aseguramiento de la calidad de la educación superior, las universidades deben ser cada vez más competitivas. Es imprescindible disponer de herramientas que permita realizar la evaluación de la productividad científica, con la finalidad de valorar objetivamente la tendencia de las universidades y si las acciones que se toman permiten mejorar la competitividad global en los rankings nacionales e internacionales.

2) La producción científica es una forma tangible y real de medir la experiencia científica y la competencia en investigación, este se convierte en un indicador valioso dentro del proceso de acreditación universitaria; sin embargo, los hallazgos ponen en manifiesto que menos de la mitad de los docentes han escrito y publicados sus obras. El bajo índice de publicación científica-académica de libros se debe al desconocimiento del proceso de maquetación y publicación; no obstante, el interés de los docentes por capacitarse en este tema es evidente; la institución debe implementar un plan integral de capacitación sobre maquetación a fin de facilitar la publicación de la producción científica docente de la Universidad Técnica del Norte y contribuir de manera significativa en la mejora de los indicadores de evaluación institucional.

3) A la hora de elegir el formato de publicación del libro (físico o electrónico), los docentes manifiestan que el crecimiento del uso y la progresiva introducción de los libros electrónicos en el ámbito académico y científico es una realidad, las razones de este avance se debe a la facilidad de acceso, inmediatez de la consulta, bajo costes de distribución, capacidad de almacenamiento, portabilidad, contribución al cuidado del medio ambiente, entre otros. Estos aspectos contribuyen de manera directa al desarrollo investigativo de las IES. En este sentido y debido a la crisis económica que atraviesa el país se cree que libros electrónicos llegarán a imponerse sobre los libros en papel.

4) Con la finalidad de que el docente optimice el tiempo y trabajo en la elaboración de su texto se recomienda considerar los aspectos detallados en este artículo a más de que el libro pase por filtros de revisión de pares y corrección de estilo ya que la eliminación o inclusión de párrafos descuadraría todo el trabajo de composición digital.

\section{REFERENCIAS}

Albornoz, M. y L. Osorio, Uso público de la información: el caso de los rankings de universidades, Revista lberoamericana de Ciencia, Tecnología y Sociedad, 12(36), 11-49 (2017)

Aithal, P., How to Increase Research Productivity in Higher Educational Institutions-IMS Model, International Journal of Scientific Research and Modern Education, 1(1), 447-458 (2016)

Acevedo, D., P. Montero y M. Duran, Análisis de la Productividad Académica de Profesores del Área de Ingeniería, doi:10.4067/S0718-50062016000200010, Formación Universitaria, 9(2), 89-96 (2016)

Altbach, P., Peripheries and centers: Research universities in developing countries, Asia Pacific Education Review, 10(1), 15-27 (2009)

Basantes, A., M. Naranjo, M. Gallegos y N. Benítez, Los Dispositivos Móviles en el Proceso de Aprendizaje de la Facultad de Educación Ciencia y Tecnología de la Universidad Técnica del Norte de Ecuador, doi: 10.4067/S071850062017000200009, Formación Universitaria, 10(2), 79-88 (2017)

Cámara ecuatoriana del libro - Núcleo de Pichincha, El libro en Ecuador, Estadísticas y Fatos ISBN (2015) 
Carrillo-Vargas, E., Academic production of the college teachers and its relationship with the communicative competence, Atenas Revista Científica de Psicología, 4(28), 75-88 (2014)

Christianson, M., A. Wiklund, A., Hammarström y B. Lundman, Sex and gender traps and springboards: a focus group study among gender researchers in medicine and health sciences, doi: 10.1080/07399332.2011.645970, Health Care Women Int, 33(8), 739-55 (2012)

CEAACES, Modelo genérico de evaluación del entorno de aprendizaje de carreras presenciales y semipresenciales de las universidades y escuelas politécnicas del Ecuador, CEAACES, Quito (2015)

Cordón-García, J., J. Alonso-Arévalo, R. Gómez-Díaz y D. Linder, Social reading: platforms, applications, clouds and tags, 1aㅡ. Edsevier (2013)

Dávila, M., Rankings universitarios internacionales y conflictos por la regulación de la educación superior, Revista Iberoamericana de Ciencia, Tecnología y Sociedad-CTS, 13(37), 67-84 (2018)

Enders, J., The academic arms race: international rankings and global competence for world class universities, Revista de la educación superior, 44(176), 83-109 (2015)

Herrera, A., Notas sobre Psicometría, Bogotá, Universidad Nacional de Colombia (1998)

Lebeau, Y., R. Stumpf y otros tres autores, Who shall pay for the public good? Comparative trends in the funding crisis of public higher education, Compare: A Journal of Comparative and International Education, 42(1), 137-157 (2012)

LOES, Ley Orgánica de Educación Superior, Presidencia de la República, Quito, Ecuador (2010)

Lux, M. y A. Pérez, Reflections on the Production, Circulation and Use of Academic Publications in the Social Sciences. Anuario Colombiano de Historia Social y de la Cultura, 44(1), 125-143 (2017)

Martínez, F., Los rankings de universidades: una visión crítica, Revista de la educación superior, 40(157), 77-97 (2011)

McCarren, M. y S. Goldman, Research leadership and investigators: gender distribution in the Federal Government, The American journal of medicine, 125(8), 811-816 (2012)

Meza, P., Estrategias de posicionamiento del Autor en Artículos de Investigación de Ciencias Sociales, Humanidades e Ingeniería: Novatos versus Expertos, doi: 10.4067/S0718-07642018000200003, Información Tecnológica, 29(2), 3-18 (2018)

Nian, L. y C. Ying, The Academic Ranking of World Universities, doi: 10.1080/03797720500260116, Higher Education in Europe, 30(2), 127-136 (2011)

Ordorika, I., Rankings universitarios, Revista de la educación superior, 44(173), 7-9 (2015)

Pérez, C., Contra los rankings de universidades: el marketing pretencioso, Revista iberoamericana de ciencia tecnología y sociedad, 10(1), 75-77 (2015)

Prasetya, D., T. Widiyaningtyas, M. Arifin y W. Sakti, Design reflowable digital book template, doi: 10.1063/1.5003506, AIP Publishing, 1887(1), 1-7 (2017)

Rodríguez-Ponce, E., W. Cohen-Hornickel y otros tres autores, Knowledge management and post-graduate teaching quality in universities: An exploratory study, Innovar, 24(52), 59-66 (2014)

Tirado, M., Maquetación de productos Editoriales, ARGG0110, IC Editorial (2016)

Torres-Velandia, S. y K. Jaimes-Cruz, Producción de conocimiento mediado por TIC: cuerpos académicos de tres universidades públicas estatales de México, Sinéctica, 44(1), 1-16 (2015)

Valderrama, J., Principales Aspectos sobre la Preparación de un Artículo para ser Publicado en una Revista Internacional de Corriente Principal, doi: 10.4067/S0718-07642005000200002, Inf. Tecnol, 16(2), 3-14 (2005)

Valencia, A. y M. Cázares, Academic and research networks management: challenges for higher education institutions in Mexico, doi: 10.1186/s41239-016-0013-2, International J. of Educational Technology in Higher Education, 13(1), 1-11 (2016)

Wang, Q., Q. Wang y N. Liu, Building world-class universities in China: Shanghai Jiao Tong University. The road to academic excellence, The making of world-class research universities, 1르. Ed., 33-62, Washington (2011)

Zornic, N., L. Bornmann y otros cuatro autores, Ranking institutions within a university based on their scientific performance: A percentile-based approach, El profesional de la información, 24(5), 551-566 (2015) 
